\title{
Splicing factor SF3B1 promotes endometrial cancer progression via regulating KSR2 RNA maturation
}

\author{
Pooja Popli ${ }^{1}$, Megan M. Richters ${ }^{2,3}$, Sangappa B. Chadchan' ${ }^{1}$, Tae Hoon Kim ${ }^{4}$, Eric Tycksen ${ }^{3}$, Obi Griffith ${ }^{2,3,5,6}$, \\ Premal H. Thaker ${ }^{7}$, Malachi Griffith $\mathbb{1}^{2,3,5,6}$ and Ramakrishna Kommagani id ${ }^{1}$
}

\begin{abstract}
Although endometrial cancer is the most common cancer of the female reproductive tract, we have little understanding of what controls endometrial cancer beyond the transcriptional effects of steroid hormones such as estrogen. As a result, we have limited therapeutic options for the $\sim 62,000$ women diagnosed with endometrial cancer each year in the United States. Here, in an attempt to identify new prognostic and therapeutic targets, we focused on a new area for this cancer-alternative mRNA splicing - and investigated whether splicing factor, SF3B1, plays an important role in endometrial cancer pathogenesis. Using a tissue microarray, we found that human endometrial tumors expressed more SF3B1 protein than non-cancerous tissues. Furthermore, SF3B1 knockdown reduced in vitro proliferation, migration, and invasion of the endometrial cancer cell lines Ishikawa and AN3CA. Similarly, the SF3B1 inhibitor, Pladienolide-B (PLAD-B), reduced the Ishikawa and AN3CA cell proliferation and invasion in vitro. Moreover, PLAD-B reduced tumor growth in an orthotopic endometrial cancer mouse model. Using RNA-Seq approach, we identified 2000 differentially expressed genes (DEGs) with SF3B1 knockdown in endometrial cancer cells. Additionally, alternative splicing (AS) events analysis revealed that SF3B1 depletion led to alteration in multiple categories of AS events including alternative exon skipping (ES), transcript start site usage (TSS), and transcript termination site (TTS) usage. Subsequently, bioinformatics analysis showed KSR2 as a potential candidate for SF3B1-mediated functions in endometrial cancer. Specifically, loss of SF3B1 led to decrease in KSR2 expression, owing to reduced maturation of KSR2 pre-mRNA to a mature RNA. Importantly, we found rescuing the KSR2 expression with SF3B1 knockdown partially restored the cell growth of endometrial cancer cells. Taken together, our data suggest that SF3B1 plays a crucial oncogenic role in the tumorigenesis of endometrial cancer and hence may support the development of SF3B1 inhibitors to treat this disease.
\end{abstract}

\section{Introduction}

In 2020, endometrial cancer is expected to be newly diagnosed in approximately 65,620 patients, and 12,590 women are predicted to die from this disease in the United States, making it the most common gynecologic malignancy ${ }^{1}$. With the rise of obesity-a strong risk factor for endometrial cancer-the number of cases are expected

\footnotetext{
Correspondence: Ramakrishna Kommagani (kommagani@wustl.edu) ${ }^{1}$ Department of Obstetrics and Gynecology, Center for Reproductive Health Sciences, Washington University School of Medicine, St. Louis, MO 63110, USA ${ }^{2}$ Division of Oncology, Department of Medicine, Washington University School of Medicine, St. Louis, MO 63110, USA

Full list of author information is available at the end of the article Edited by $\mathrm{G}$. Blandino
}

to double by $2030^{2}$. Although most endometrial cancer patients present with low-grade, estrogen-driven, type 1, early-stage disease and have a favorable prognosis, those with advanced-stage, hormone-independent, type 2, metastatic disease have few treatment options and poor survival outcomes. Currently, major treatment options include endocrine therapy, chemotherapy, immunotherapy, radiotherapy, and hysterectomy. However, endocrine therapy is not widely used because of its limited efficacy, and hysterectomy is not a suitable option for women who wish to preserve fertility. Developing new therapeutic options will require a greater understanding of genes that contribute to endometrial cancer progression. 
Recent work in several cancers has revealed that dysregulation of splicing factors can lead to the generation of abnormal mature transcripts ${ }^{3}$ that drive tumorigenesis ${ }^{4-7}$. For example, mutations in U2AF1, SRSF2, ZRSR2, and $S F 3 B 1$ have been identified in myelodysplastic syndromes, chronic lymphocytic leukemia, uveal melanoma, and breast cancer $^{8-19}$. In endometrial cancer, the SF3B1 is one of the frequently mutated splicing factor ${ }^{20,21}$. SF3B1 is a core component of the $\mathrm{U} 2$ small nuclear ribonucleoprotein, which specifically recognizes the $3^{\prime}$ splice site at intron-exon junctions ${ }^{22,23}$. In endometrial cancer and several other cancers, SF3B1 appears to act as an oncogene, as most of the mutations occur in hotspots ${ }^{20}$. Besides, overexpression of SF3B1 has also been reported to drive tumorigenesis in several cancers, including breast cancer, prostate cancer, and myelodysplastic syndromes $^{24-26}$. Moreover, a recent study revealed that knock down of overexpressed SF3B1 expression reduced breast cancer cell proliferation, migration, and invasion ${ }^{26}$. However, whether SF3B1 overexpression likewise promotes endometrial cancer progression is unknown.

Here, we report that SF3B1 protein is overexpressed in human endometrial tumor samples and endometrial cancer cell lines. Further, we show that knockdown of SF3B1 or treatment with the SF3B1 inhibitor Pladienolide- $B$ reduces cell viability, migration, and invasion of endometrial cancer cells in vitro and endometrial tumor cell growth in vivo. Finally, we report that knockdown of SF3B1 alters mRNA maturation of the kinase suppressor of Ras gene KSR2 and KSR2 acts as a downstream mediator of SF3B1 function(s) in endometrial cancer. Thus, SF3B1 protein expression may be a prognostic biomarker and a therapeutic target for treating endometrial cancer patients.

\section{Materials and methods}

Mouse and human study approval

Animal studies were performed according to a protocol (\#20160227) approved by the Institutional Animal Care and Use Committee of Washington University School of Medicine, Saint Louis, MO, USA. Human volunteers provided written informed consent in accordance with an Institutional Review Board-approved protocol (\#201612127) from Washington University School of Medicine and the guidelines of the Declaration of Helsinki.

\section{Cell culture}

The human endometrial cancer cell lines Ishikawa (Sigma, St. Louis, MO) and AN3CA, KLE, and RL-95-2 (all from American Type Culture Collection) were purchased and used within 6-8 months. All cells were maintained in MEM/DMEM medium supplemented with $10 \%$ fetal bovine serum at $37^{\circ} \mathrm{C}$ and $5 \% \mathrm{CO}_{2}$.

\section{Orthotopic endometrial cancer model}

Female athymic nude mice (6-8 weeks of age; Jackson Laboratory) were maintained under pathogen-free conditions with food and water provided ad libitum.

The sample size of animal experiment was chosen based on the preliminary experiments and similar well-designed experiments, and no statistical method was used. Investigators were blinded to the treatment groups during data collection and subsequent data analysis. Before surgery, animals received intramuscular $0.1 \mathrm{mg} / \mathrm{kg}$ buprenorphine hydrochloride (Temgesic, Reckitt Benckiser, Berkshire, UK) for analgesia. Mice were anesthetized with $100 \mathrm{mg} / \mathrm{kg}$ ketamine and $5 \mathrm{mg} / \mathrm{kg}$ xylazine and placed on a heating pad in dorsal decubitus. Their dorsal skin was cleaned and sterilized with betadine, and a $0.5-\mathrm{cm}$ incision was made in the right lower flank to expose the right uterine horn. A single-cell suspension of $50 \mu \mathrm{l}$ of ice-cold Matrigel (BD Matrigel Basement Membrane Matrix, BD Biosciences, San Jose, CA) containing $2 \times 10^{6}$ AN3CA cells was injected into the endometrial cavity through the myometrium by using a $0.3 \mathrm{~mm}$ insulin syringe (Omnican 50; B-Braun, Melsungen, Germany). The needle was retracted after waiting for a few seconds to allow the Matrigel cell suspension to polymerize and to prevent leakage into the abdominal cavity. The uterine horn was returned to its original position, and the incision was closed with staples. After the surgery, animals were placed in a warm environment and supervised until full recovery. Three weeks later, mice were randomly assigned to receive either $10 \mathrm{mg} / \mathrm{kg}$ PLAD-B $(n=4)$ or vehicle (DMSO) $(n=4)$ intraperitoneally on days $0,2,4$, and 6 . Animals were weighed weekly. Two weeks after the last dose, the mice were sacrificed, and the tumors were imaged, weighed, and processed for histological analysis and immunofluorescence.

\section{Co-transfection experiment}

Lipofectamine 2000 (Invitrogen) was used to cotransfect Ishikawa cells with SF3B1 siRNA or Control siRNA and pEGFPC1-hKSR2 or pEGFPC1-empty vector. After $36 \mathrm{~h}$, cells were counted and re-plated in 96-well plates, and the relative proliferation rate was evaluated with the MTT proliferation kit at 0,24 , and $48 \mathrm{~h}$. Experiments were performed in triplicates.

\section{Statistics}

A two-tailed paired Student's $t$-test was used to test for statistical significance, defined as $P<0.05$. One-way analysis of variance (ANOVA) was used for multiple group comparisons. Asterisks represent the level of significance: "P $P=0.05,{ }^{* *} P=0.01,{ }^{* * *} P=0.001$, and ${ }^{* * * *} P=0.0001$.

A detailed description of the materials and methods used in this study is available in the online Supplementary Material. 


\section{Results}

SF3B1 expression is elevated in endometrial cancer

To investigate whether SF3B1 was involved in endometrial tumorigenesis, we first examined its expression in a tissue microarray containing 102 human endometrial cancer samples of various tumor grades. SF3B1 expression was higher in grades I, II, and III endometrial tumors than in normal endometrial tissues (Fig. 1a, b). Importantly, we observed a grade-dependent increase in SF3B1 expression with the highest expression in Grade III tumors. The distribution of cases with higher SF3B1 expression as follows: Grade I, 26/34 (76\%), Grade II, 34/36 (94\%), and Grade III, 27/27 (100\%). Consistent with this, we found that tumors in a genetic mouse model of endometrial cancer $\left(P R^{\text {Cre } /+}\right.$ Pten $\left.^{\text {flox/flox }}\right)$ overexpress SF3B1 protein (Fig. 1c). Next, we compared SF3B1 mRNA and protein expression in primary human endometrial epithelial cells (our experimental control) and three Type 1 endometrial cancer cell lines: Ishikawa cells (isolated from a welldifferentiated tumor), RL-95-2 cells (from a moderately differentiated tumor), and AN3CA cells (from a metastatic undifferentiated tumor). Additionally, we examined SF3B1 expression in one Type II endometrial cancer cell line, KLE (from a poorly differentiated tumor). Although SF3B1 mRNA was only elevated in KLE cells, SF3B1 protein was more highly expressed in Ishikawa, AN3CA, and KLE cells than in control cells (Fig. 1d). Importantly, we performed full exon sequencing of $S F 3 B 1$ in Ishikawa, AN3CA, and RL-95-2 cells (data not shown) and found no SF3B1 missense mutations in any of these cell lines. These data indicate that endometrial cancer cell lines overexpress SF3B1 protein in the absence of mutations or elevated mRNA overexpression. Together, these results demonstrate that SF3B1 expression is elevated in endometrial cancer at protein level.

\section{SF3B1 is required for endometrial cancer cell proliferation} and cell cycle progression

Given that SF3B1 expression was elevated in endometrial cancers, we wondered whether SF3B1 promotes endometrial cancer cell proliferation. To investigate this, we transiently transfected Ishikawa and AN3CA cells (the cell lines in which SF3B1 was most highly expressed) with control or SF3B1-targeted small interfering RNA (siRNA). Western blotting and quantitative real-time PCR (Fig. 2a, d) confirmed that the siRNA substantially reduced SF3B1 protein and mRNA expression. After $48 \mathrm{~h}$, we re-plated the cells and followed proliferation for $72 \mathrm{~h}$ and found that SF3B1 siRNA significantly decreased the proliferation of Ishikawa and AN3CA cells (Fig. 2b, e). In addition, clonogenic assays revealed that SF3B1 knockdown reduced Ishikawa cells proliferation by $84 \%$ and AN3CA

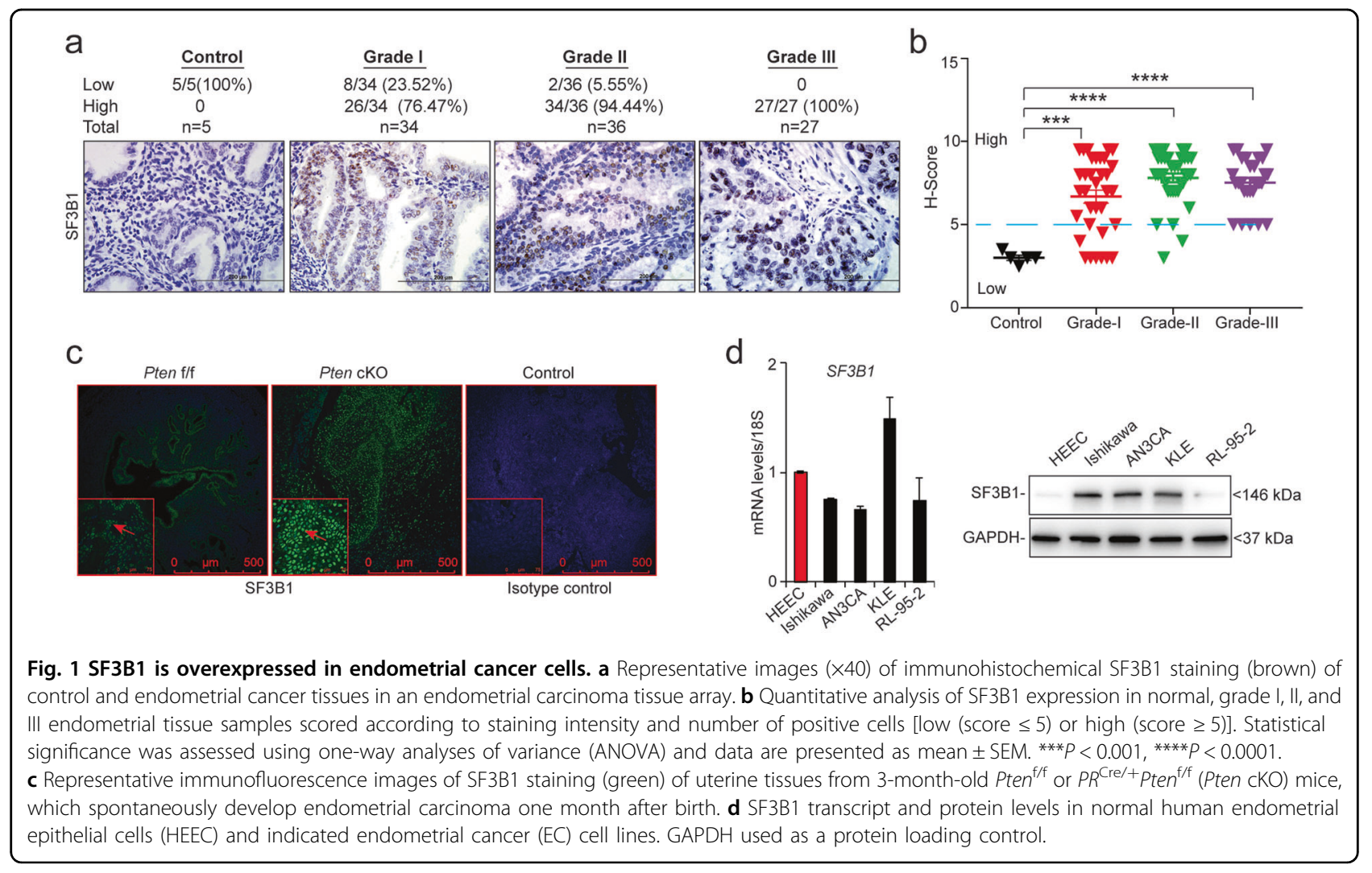




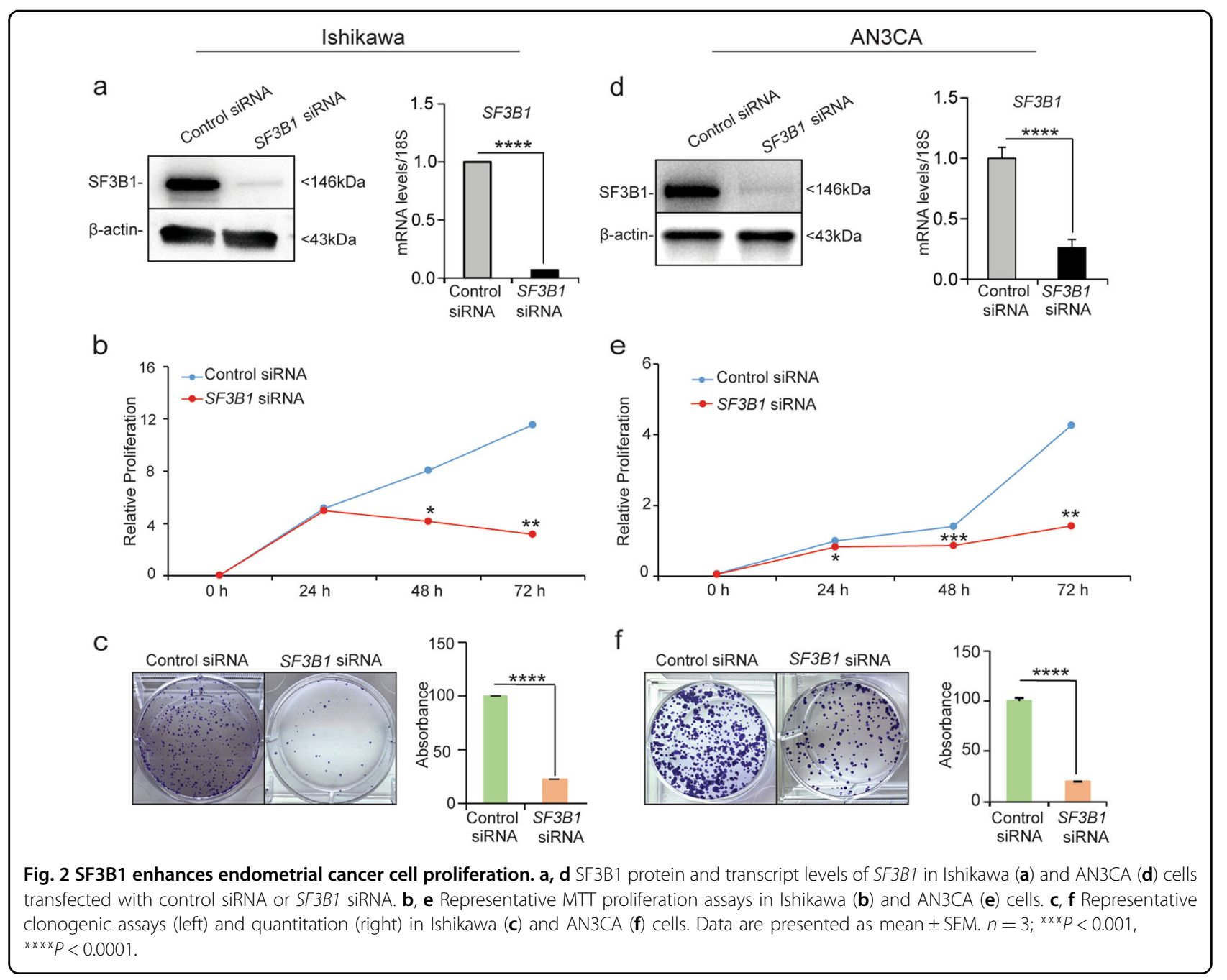

cell proliferation by $81 \%$ (Fig. $2 \mathrm{c}, \mathrm{f}$ ). These results suggested that SF3B1 promotes endometrial cancer cell proliferation.

Next, we evaluated the effect of SF3B1 knockdown on cell cycle progression. Flow cytometry analysis revealed that, compared to cells transfected with control siRNA, a much greater proportion of Ishikawa and AN3CA cells in which SF3B1 was knocked down were arrested in the G2/ $M$ phase of the cell cycle, with a concomitant reduction in the percentage of cells in the G1 phase (Fig. 3a, b, d, e). These results were consistent with findings of Dolatshed et al. $^{25}$ that SF3B1 knockdown caused G2/M cell cycle arrest in hematopoietic stem and progenitor cells in myelodysplastic syndrome. Next, we examined the expression of the cell cycle regulatory proteins: Cyclin D1 (required throughout the cycle), Cyclin E1 (S-phase transition), Cyclin B1 (transition from G2 to M), p21 (cell cycle inhibitor), CDK4 (progression through G1 phase), and CDK2 (progression through G1 phase) ${ }^{27}$. Expression of Cyclins B1, E1, and D1 was efficiently reduced in cells in which SF3B1 was knocked down, whereas the expression of p21 was elevated (Fig. 3c, f). These results suggested that SF3B1 silencing reduced Ishikawa and AN3CA cell proliferation by inducing $\mathrm{G} 2 / \mathrm{M}$ cell cycle arrest.

\section{SF3B1 is required for tumor cell migration and invasion}

Metastasis, which occurs after cells undergo the epithelial-to-mesenchymal transition (EMT), is the leading cause of most cancer-related deaths ${ }^{28-31}$. To undergo EMT, tumor cells must reduce their expression of E-cadherin and increase the expression of vimentin to become capable of migrating and invading other tissues ${ }^{28-31}$. To determine whether SF3B1 was required for tumor cell migration, we transfected Ishikawa and AN3CA cells with SF3B1 siRNA or control siRNA, confirmed that SF3B1 mRNA expression was efficiently knocked down (Fig. 4a, e), and then performed wound-healing scratch assays. At $48 \mathrm{~h}$ post-scratch, Ishikawa and AN3CA cells expressing SF3B1 siRNA were significantly less migratory than cells expressing control siRNA (Fig. 4b, f). The migration capacity of SF3B1 

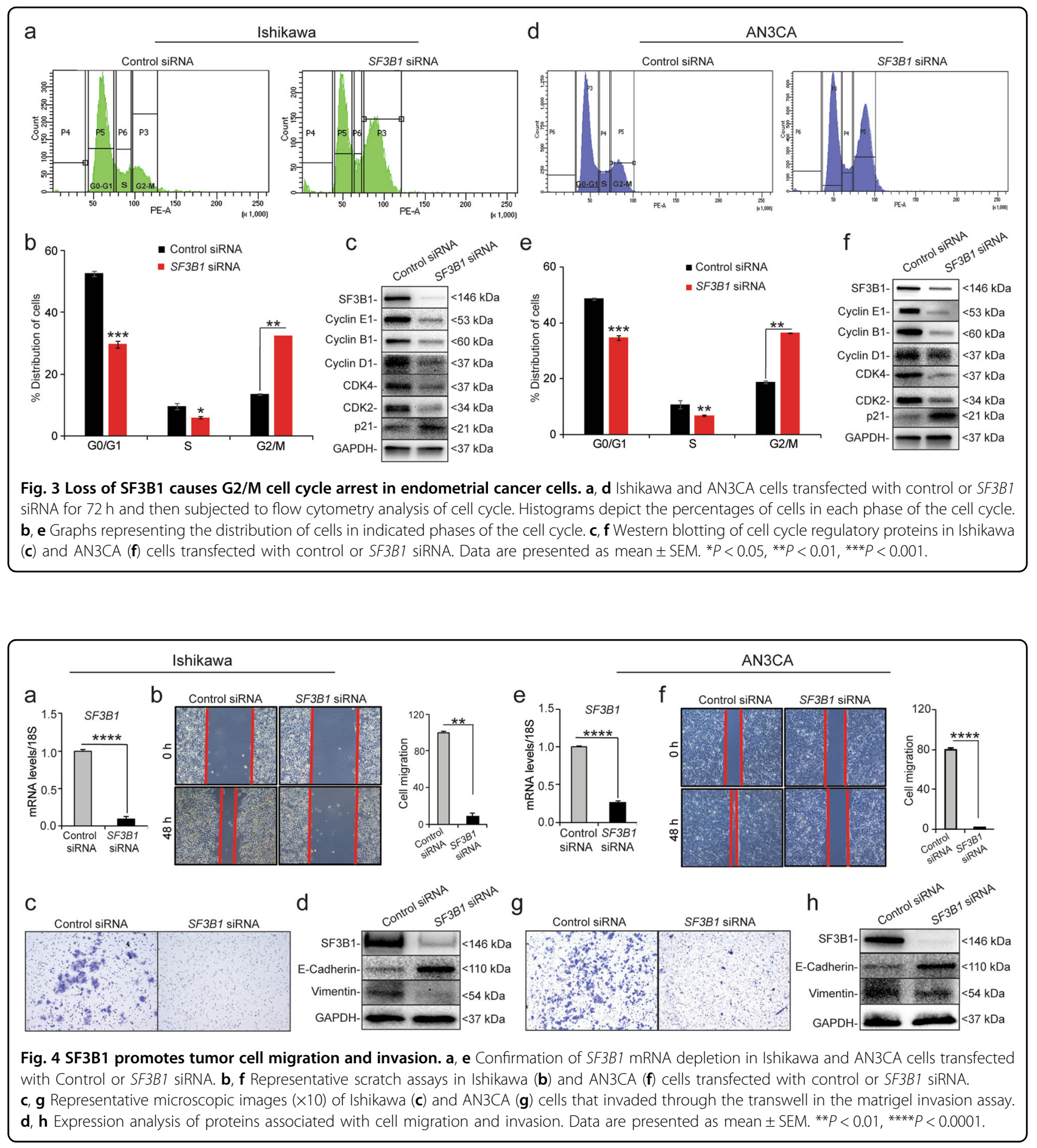

depleted Ishikawa and AN3CA when calculated was 70-80\% less than their control siRNA cells respectively (Fig. 4b, f).

To evaluate whether SF3B1 was required for invasion, we performed an assay in which cells transfected with SF3B1 siRNA or Control siRNA were placed into the upper chamber of matrigel-coated transwells. We then stained the cells that invaded through the matrigel into the lower chamber and found that, compared to cells transfected with control siRNA, fewer cells transfected with SF3B1 siRNA were able to invade through matrigel (Fig. 4c, g). Next, we examined the expression of adhesion markers: E-cadherin and vimentin associated with EMT. We found that cells in which SF3B1 was knocked down had higher 
E-cadherin expression and lower vimentin expression than control siRNA-treated cells (Fig. 4d, h). Together, these results suggest that SF3B1 promotes migration and invasion behaviors of endometrial cancer cells.

\section{The SF3B1 inhibitor Pladienolide-B reduces endometrial cancer cell proliferation, migration, and invasion in vitro}

PLAD-B is a macrocyclic lactone originally obtained from Streptomyces platensis Mer-11107, strain isolated from a soil sample collected in Kanagawa, Japan (accession number FERM P-18144; Bioconsortia Program Laboratory National Institute of Advanced Industrial Science and Technology, Japan) ${ }^{32-34}$. PLAD-B is spliceosome inhibitor that specifically targets the SF3B1 spliceosome subunit ${ }^{35}$ and has anti-tumor activity in multiple cancers ${ }^{35,36}$. For example, when immunocompromised mice were implanted with primary cultured cells from gastric cancer patients, the tumors regressed with PLAD-B treatment ${ }^{36}$. Thus, we determined the effect of PLAD-B on in vitro proliferation, migration, and invasion of endometrial cancer cells. Both Ishikawa and AN3CA cells were treated with vehicle or increasing concentrations of PLAD-B, and cell proliferation was measured $0,24,48$, and $72 \mathrm{~h}$ later. As expected, PLAD-B substantially decreased the proliferation of Ishikawa and AN3CA cells in a dose-dependent manner with $\mathrm{IC}_{50}$ of $\sim 10 \mathrm{nM}(p<0.01)$ (Fig. 5a, e). Similarly, in clonogenic assays, PLAD-B substantially reduced the clonal growth of Ishikawa and AN3CA cells (Fig. 5b, f). Next, we examined the effect of PLAD-B on cell migration and invasion. Scratch assays revealed that PLAD-B reduced the migration of Ishikawa cells by $60 \%$ and of AN3CA cells by $80 \%$ (Fig. 5c, g). In the invasion assay, Ishikawa and AN3CA cells treated with PLAD-B failed to invade through matrigel (Fig. 5d, h). These data indicate that PLAD-B efficiently reduces the oncogenic potential of SF3B1 in endometrial cancer cells. Additionally, we also evaluated the effect of SF3B1 knockdown on KLE cells viability, migration, and cell cycle progression and found the similar effects as we observed in Ishikawa and AN3CA cells (Fig. S1). Moreover, consistent with minimal SF3B1 protein levels, RL-95-2 cells showed diminished impact of SF3B1 knockdown or PLAD-B treatment on cell viability and migration in comparison to other three endometrial cancer cells that have robust SF3B1 protein levels (Fig. S2). Importantly, whereas SF3B1-depleted RL-95-2 cells are in a growing phase still at $72 \mathrm{~h}$, three other endometrial cancer cell lines with high SF3B1 levels were in diminished cell growth state (Fig. S2).

\section{PLAD-B inhibits endometrial tumor growth in vivo}

We next sought to assess the activity of PLAD-B in vivo in an orthotopic model of endometrial cancer. AN3CA cells $\left(2 \times 10^{6}\right)$ were injected into the right uterine horns of mice, leaving the left uterine horns untreated as controls.
After 3 weeks, mice were randomized to receive either vehicle (DMSO) or $10 \mathrm{mg} / \mathrm{kg}$ PLAD-B intraperitoneally every other day for 1 week $^{36}$. Two weeks later, mice were euthanized and uteri were collected (Fig. 6a). In the vehicle group, the tumor-cell-injected uterine horns were $\sim 10$ times larger than the control uterine horns (Fig. 6b, left images). Histological evaluation revealed normal histology with intact endometrial glands in the left uterine horn and a solid growing primary tumor in the right uterine horn (Fig. 6b, left images). The tumors showed characteristics of grade 3 endometrioid endometrial cancers, with areas of necrosis and myometrial invasion. In the PLAD-B group, the control and tumor-cell-injected horns were similar in size (Fig. 6b, right images). Additionally, the tumor-cell-injected horns in the PLAD-B group had a typical uterine structure with intact endometrial glands (Fig. 6b, right images). However, we noted the remnants of regressing tumors in the right uteri of PLAD-B-treated mice (Fig. 6b, right images). Quantification analysis showed that tumor weight reduced $\sim 76 \%$ in PLAD-B-treated mice compared to vehicle-treated mice (Fig. 6b). These results suggest that PLAD-B effectively reduced the tumor growth. However, PLAD-B appeared to be non-toxic, as we did not notice any differences in behavior, feeding habits, or mobility between vehicle- and PLAD-B-treated mice (data not shown).

To begin to uncover how PLAD-B reduced tumor growth, we examined the expression of proliferation marker Ki-67. In the control uterine horns of vehicle-treated mice, Ki-67-positive cells were evident in the luminal and glandular epithelium. The tumor-cell-injected uterine horns had substantially more Ki-67-positive cells, indicating the growth of a solid tumor (Fig. 6c). The tumor-cellinjected horns in PLAD-B treated mice had substantially fewer Ki-67-positive cells than the corresponding horns in vehicle-treated mice (Fig. 6c) indicating that PLAD-B efficiently reduced tumor cell proliferation. PLAD-B also appeared to inhibit normal cell proliferation, as we saw fewer Ki-67-positive cells in the normal horns of PLAD-Btreated mice than in the corresponding horns of vehicletreated mice (Fig. 6c, right images).

We wondered whether PLAD-B affected SF3B1 expression and thus stained the uterine horns from the two groups of mice with an SF3B1-specific antibody. In the vehicle-treated mice, the tumor-cell-injected horns had substantially higher SF3B1 expression than the corresponding normal horns (Fig. 6d). SF3B1 expression was mainly restricted to the luminal and glandular epithelium in control uteri (Fig. 6d). As expected, we did not notice any difference in SF3B1 expression between the tumorcell-injected horns in the vehicle and the PLAD-B-treated mice, indicating that PLAD-B inhibits SF3B1 activity but not expression. Together, these findings suggest that SF3B1 could be targeted to reduce endometrial cancer growth. 


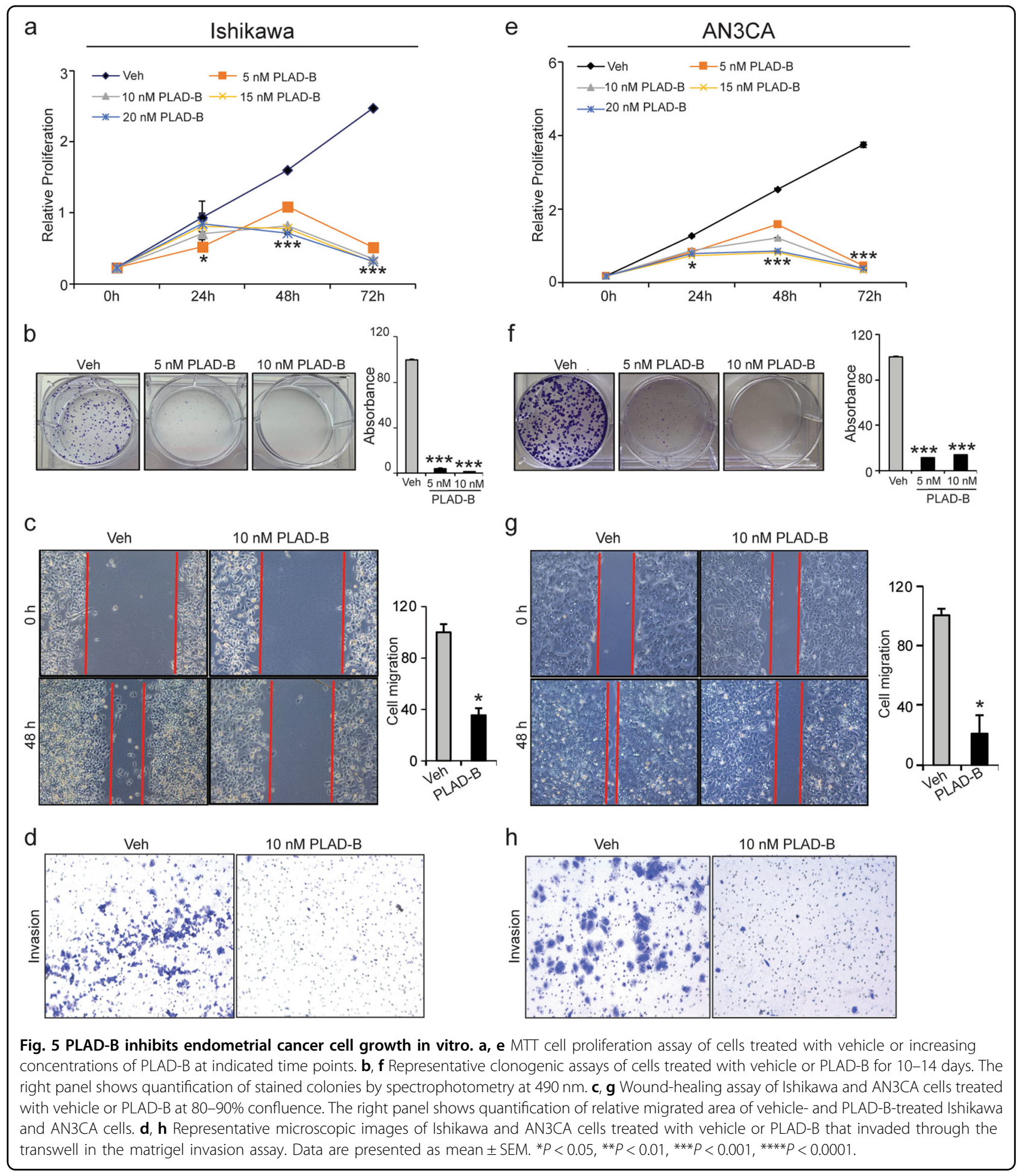

\section{Identification of SF3B1-driven transcriptome and} alternative splicing events in endometrial cancer cells

Since overexpressed SF3B1 promotes endometrial tumorigenesis both in vitro and in vivo, we next investigated the underlying mechanism. Toward this, we determined the SF3B1 transcriptome by performing RNA-seq analysis from Ishikawa cells depleted with SF3B1. As shown in Fig. 7a, hierarchical clustering revealed a distinct SF3B1-dependent transcriptome in in Ishikawa cells. Using a 2.5-fold cutoff and Benjamini-Hochberg false discovery rate (FDR) of $<0.01$ threshold for inclusion, we identified 1992 differentially expressed genes (DEGs) between 


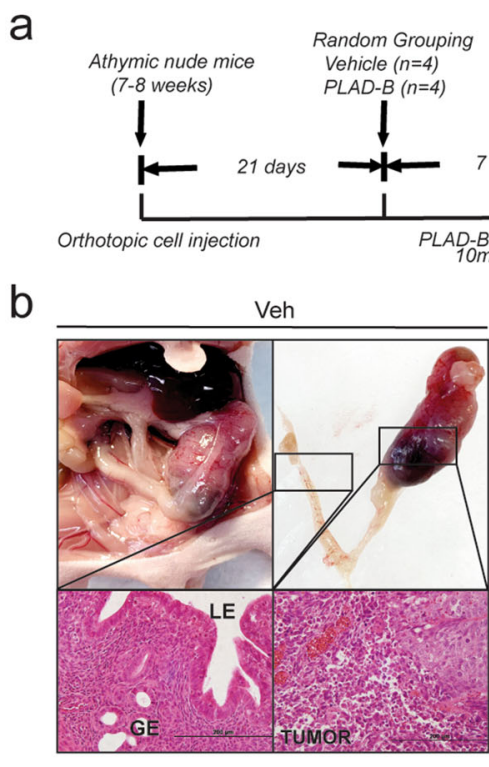

C
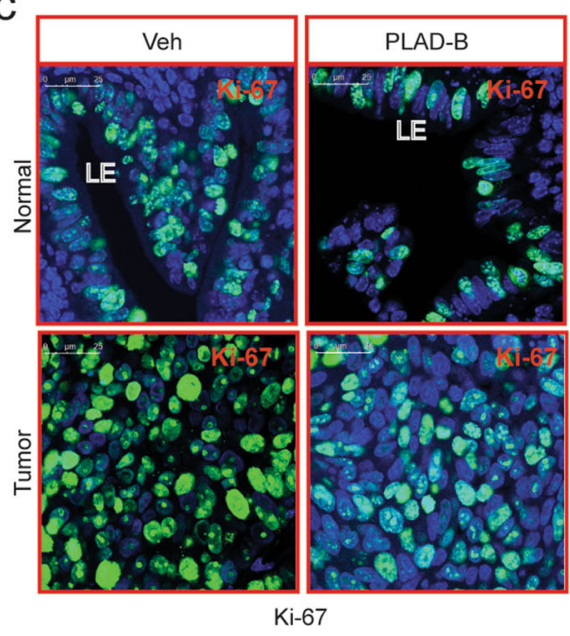

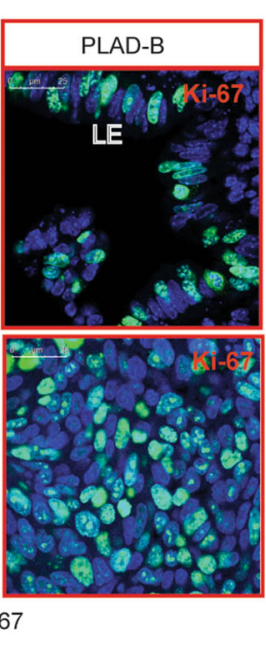

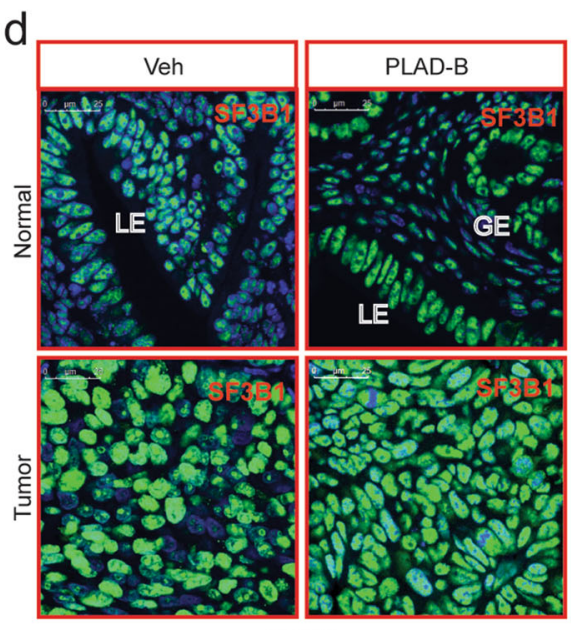

SF3B1

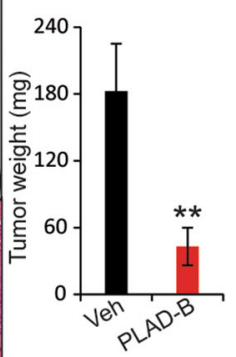

Fig. 6 PLAD-B inhibits endometrial tumor growth in vivo. a Schematic representation of experimental procedure. $\mathbf{b}$ Representative images of uteri from mice treated with vehicle or PLAD-B and H\&E-stained uterine horns from vehicle- or PLAD-B-treated animals (left). Quantification of tumor weights from vehicle- or PLAD-B-treated mice at endpoint (Right). c, d Representative confocal immunofluorescent cross-sectional images of uteri from vehicle- or PLAD-B-treated animals stained for Ki-67 and SF3B1; scale bar: $200 \mu \mathrm{m}$. LE luminal epithelia, G glandular epithelia. Normal, control uninjected horn; tumor, AN3CA cell-injected uterine horn.
Control and SF3B1-depleted Ishikawa cells (Fig. 7c and Supplementary Table 1). Further, Gene Ontology (GO) enrichment analysis revealed that SF3B1 regulated DEGs enriched in several biological processes, including cell adhesion, angiogenesis, and cytokine production involved in immune response, and ATP binding (Supplementary Table 2). Importantly, pathway analysis has been shown that much of the DEGs enriched in calcium signaling pathway, Ras signaling, and metabolic pathways (Supplementary Table 2). This enrichment analysis suggests that SF3B1 overexpression promotes pro-cancer-specific transcriptome in endometrial cancer.
Further, to gain insight into the spectrum of genes that are aberrantly spliced, we used DEXSeq to perform differential exon usage analysis of our RNA-seq data. DEXseq analyzed 6,40,456 exons for differential exon usage. A complete set of DEXseq raw counts, normalized counts, and statistics for every exon is provided as Supplementary Table 3 (annotations for each exon are provided in Supplementary Table 4). Summarization of the exons disrupted suggested that $S F 3 B 1$ depletion leads to multiple categories of alternative splicing including alternative exon skipping (ES), transcript start site, and transcript termination site usage (Fig. 7d). A total of 3840 exons exhibited 
a

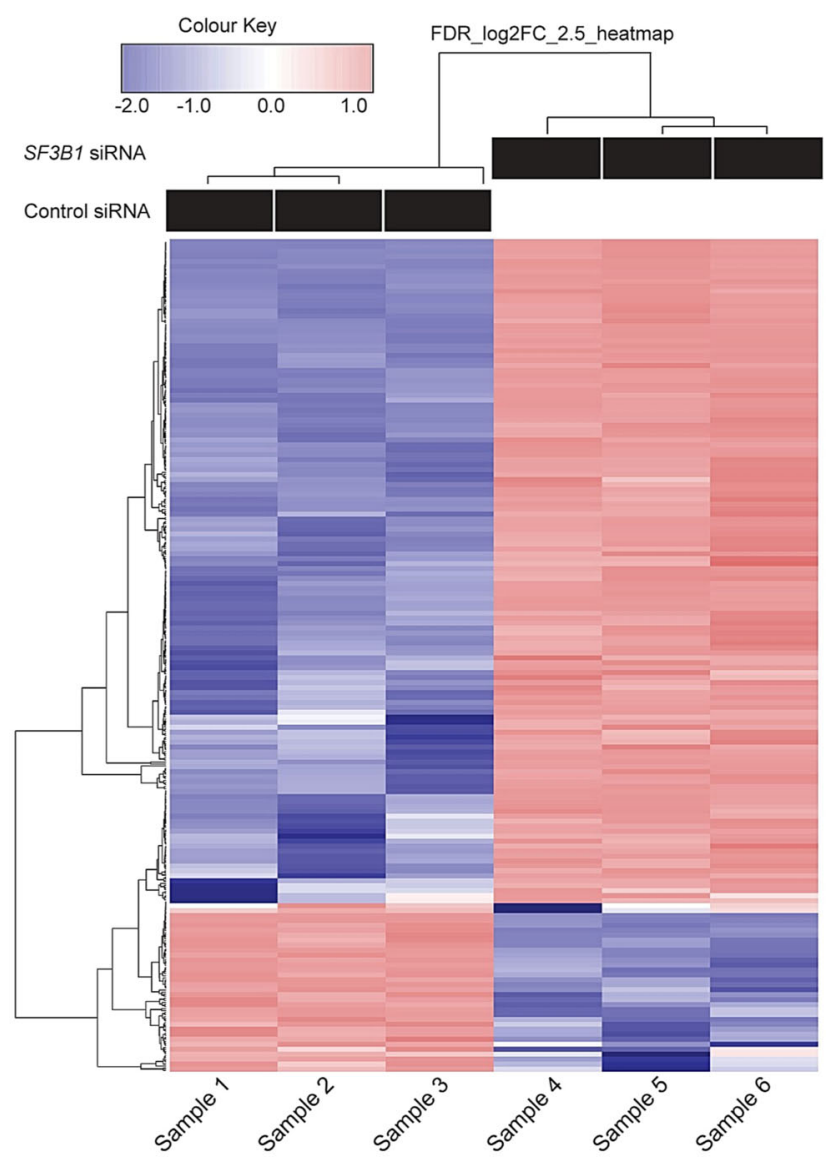

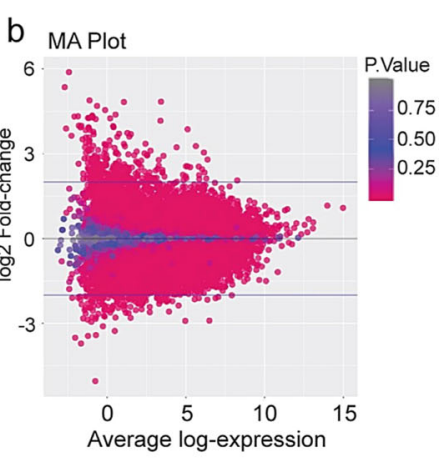

C

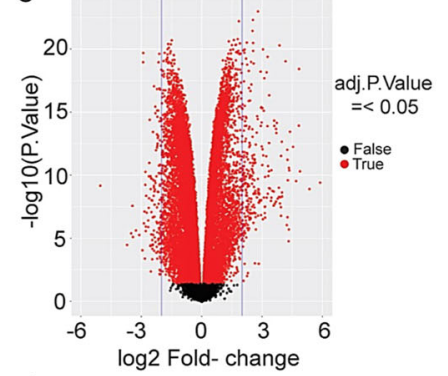

d

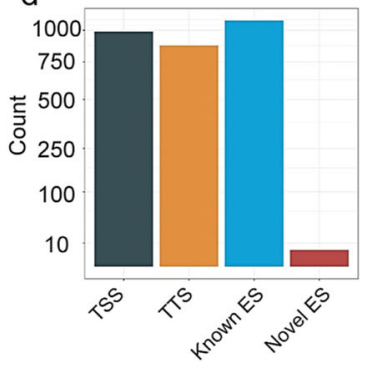

e

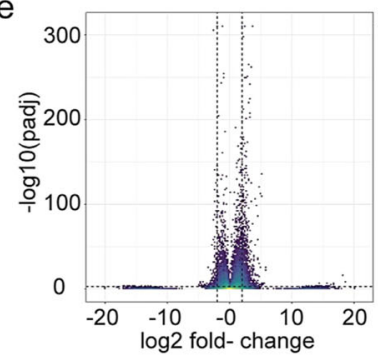

$f$
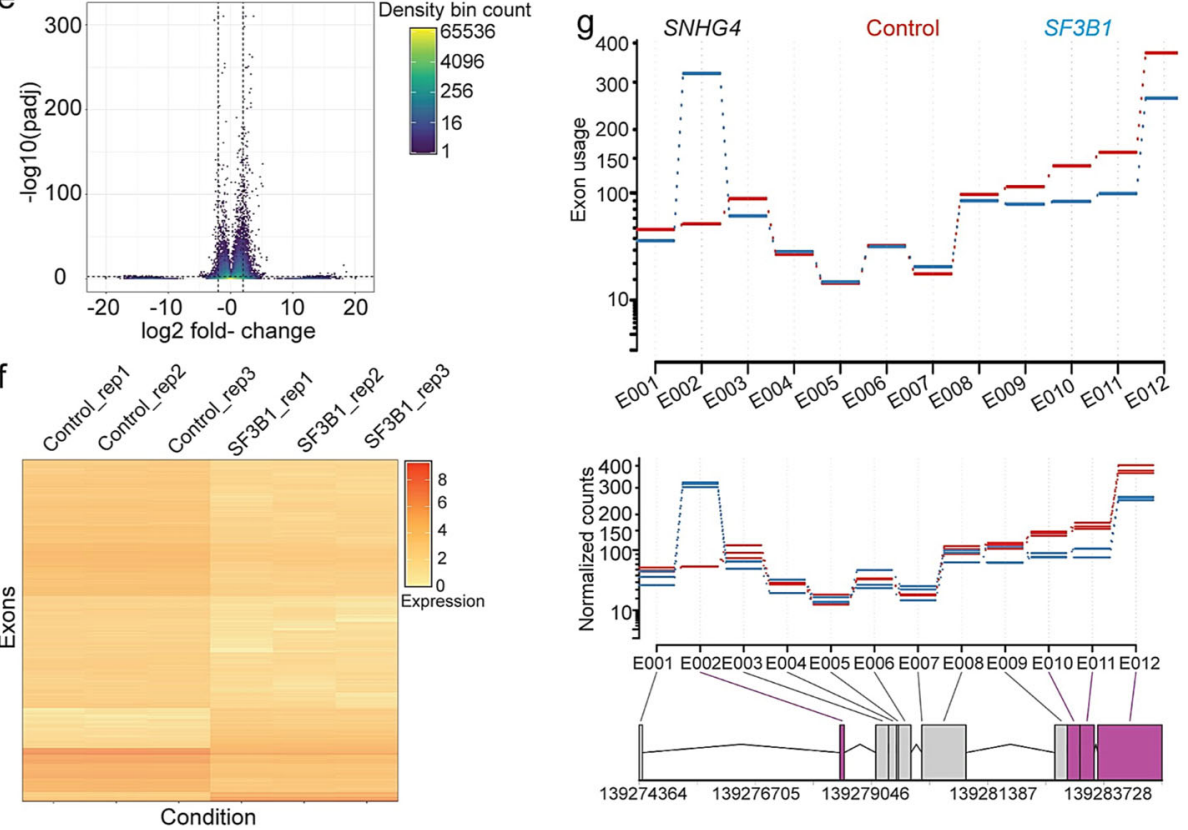

Fig. 7 (See legend on next page.) 
(see figure on previous page)

Fig. 7 SF3B1 depletion alters the transcriptome and AS events in EC cells. a Heat map of mRNAs differentially expressed between control and SF3B1 knockdown Ishikawa cells with cutoff of $F D R<0.01$ and $\log F C>2.5 ; n=3$ each group. $\mathbf{b}$ MA plot showing the appropriate normalization of RNA-seq data. c Volcano plots for all the DEGs in each comparison. The left side indicates downregulated genes and the right side indicates that upregulated DEGs with $p$ values $<0.05$. d Summary of alternative exon usage by alternative transcription category. e Volcano density plot of exons' FDR corrected adjusted $p$ value and log2 fold change of SF3B1 knockdown vs. control. Color represents the density of each bin. Dashed lines represent significance thresholds. $\mathbf{f}$ Heat map depicting normalized counts for exons with sufficient coverage. Color represents log2 $(x+1)$ transformed normalized counts. g Exon usage and normalized counts for control and SF3B1 knockdown conditions for ENSG00000281398 [SNHG4]. Color (purple) in the gene map indicates significant exons.

significant loss or gain, determined by FDR corrected adjusted $p$ value cutoff of 0.01 and minimum $\log 2$ fold change $>2$, which affected 2160 total gene loci (Fig. 7e). Unsupervised hierarchical clustering was performed on exons with sufficient coverage and variance. Replicates in the control and SF3B1 knockdown conditions clustered together tightly, indicating a consistent and predictable deregulation of normal splicing upon SF3B1 knockdown (Fig. 7f). Specific novel alternative splicing events induced by $S F 3 B 1$ were readily identifiable and ranged from relative simple alternative ES (e.g. Fig. $7 \mathrm{~g}$ ) to much more complex patterns. Overall, the alternative splicing analysis revealed wide scale disruption of alternative splicing across the transcriptome.

\section{Validation of DEGs identified by RNA sequencing}

Given the wide scale alterations across transcriptome with SF3B1 knockdown (Fig. 7a), next we aimed to find a specific DEGs that might be a candidate downstream mediators of SF3B1 action. For this, we narrowed seven DEGs based on their biological functions. Out of these seven DEGs, five (KSR2, GJD3, TRAF1, TBC1D16, and DOCK11) were downregulated and (FOXQ1, MYLK2) two were upregulated (Fig. S3). First, we validated the expression of these seven altered genes with SF3B1 knockdown in both Ishikawa and AN3CA cells (Fig. S3). Since, Ras signaling is the primary mitogenic pathway of endometrial cancer, we decided to focus on KSR2, a Ras signaling promoting gene for subsequent studies (Fig. S4). KSR2 is a molecular scaffold protein shown to be critical in the integration of various mitogenic and metabolic pathways $^{37-39}$. As expected, we found SF3B1 regulated the KSR2 expression in endometrial cancer cells both at transcript and protein levels (Fig. 8a).

\section{KSR2 contributes to SF3B1-dependent proliferation in endometrial cancer cells}

SF3B1 is an important regulator of pre-mRNA machinery ${ }^{24,40,41}$ and we found that KSR2 transcript levels were abrogated with SF3B1 knockdown (Fig. 8a). Therefore, we wondered whether SF3B1 is required for maturation of KSR2 pre-mRNA to mature mRNA. To test this, we measured mature (spliced) and immature (unspliced) KSR2 mRNAs using intron-exon and exon-exon junction-specific primers (Fig. 8b). While knockdown of SF3B1 abrogated mature KSR2 mRNAs in both Ishikawa and AN3CA cells, KSR2 pre-mRNAs remained stable in Ishikawa cells and reduced modestly in AN3CA cells (Fig. 8c). This reduced level of pre-mRNA might be due to splicing coupled transcription defect ${ }^{42}$ or due to reduced stability of pre-mRNA owing to intron retention (splicing defect) ${ }^{43}$. Importantly, the relative ratio of pre-mRNA/mRNA was significantly higher with SF3B1 knockdown in both the Ishikawa and AN3CA cells, indicating that the reduced splicing efficiency in the absence of SF3B1 lead to an accumulation of pre-mature KSR2 RNA. Finally, to determine whether KSR2 contributes to overall SF3B1 function, we performed a rescue experiment by co-transfecting the Ishikawa cells with siRNA and human KSR2 plasmid and determined the cell proliferation. As expected, KSR2 protein levels abrogated with SF3B1 knockdown restored with KSR2-GFP plasmid transfection in Ishikawa cells (Fig. 8d). Importantly, rescuing the KSR2 protein in SF3B1-depleted cells partially restored the reduced cell proliferation due to SF3B1 knockdown (Fig. 8e). Taken together, these data suggest that SF3B1 exerts its functions by generating mature splice variants and KSR2 is one such downstream effectors of SF3B1 function in endometrial cancers (Fig. 8f).

\section{Discussion}

Here, we present several pieces of evidence indicating that the splicing factor SF3B1 plays an important role in endometrial cancer pathogenesis. First, we found increased SF3B1 protein expression in human endometrial tumors and three endometrial cancer cell lines. This is consistent with multiple reports that showed an elevated expression of the other splicing factors in several human cancers ${ }^{8}$. Second, our in vitro data demonstrated that SF3B1 promotes endometrial cancer cell proliferation, cell cycle progression, migration, and invasion. Third, our in vivo data indicated that SF3B1 inhibitor, PLAD-B, reduces endometrial tumor growth in mice. Finally, we found that SF3B1 mediates pro-proliferative function partially by driving KSR2 mRNA maturation.

In our study, decreased cell viability and associated cell cycle regulators expression in SF3B1-depleted cells highlights its pro-proliferative role in endometrial cancer. 


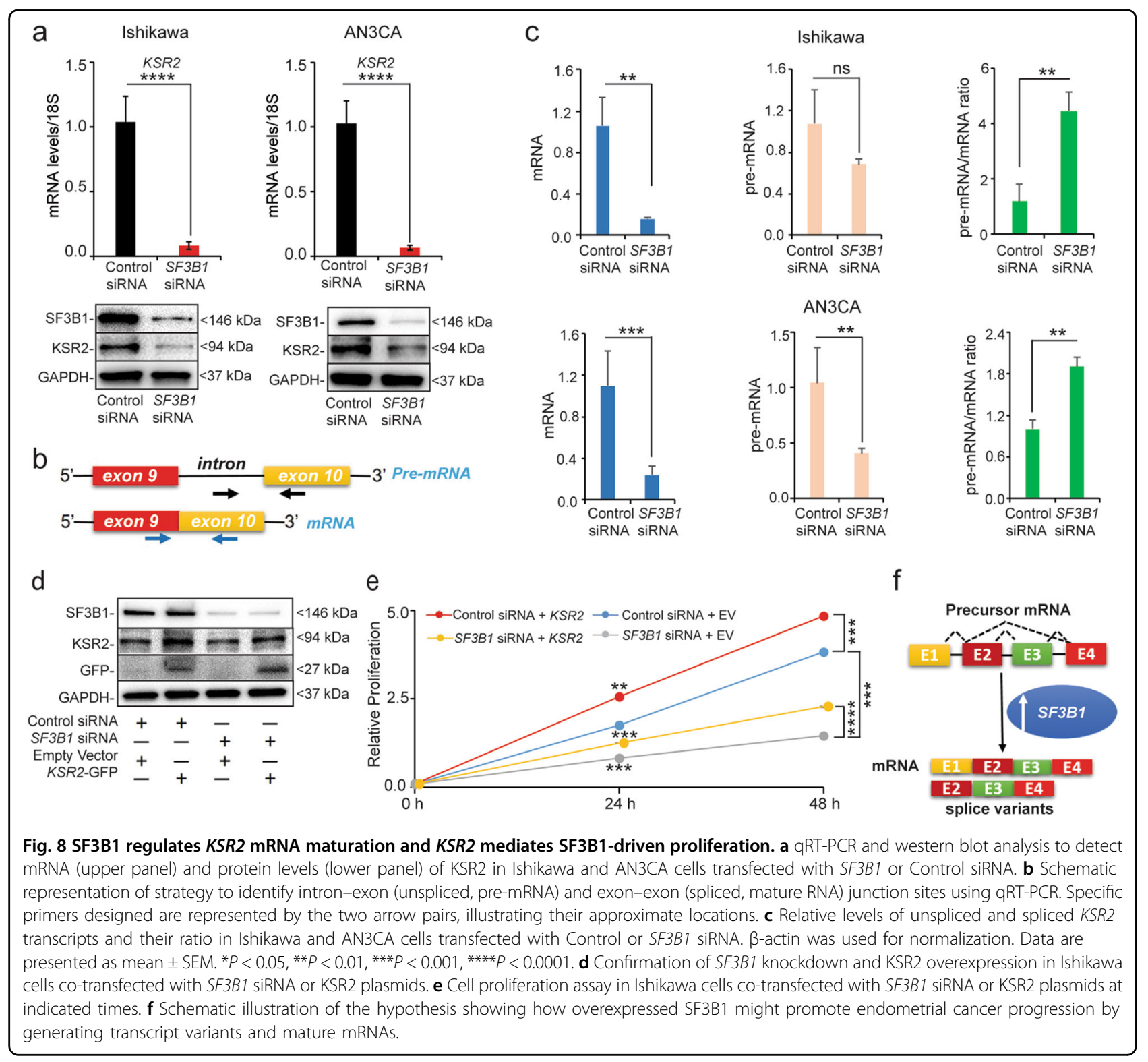

Interestingly, a recent report suggested that aberrant splicing by mutant SF3B1 altered the transcriptome, proteome, and metabolome of human cells and caused mis-splicing of various associated metabolic genes ${ }^{44}$. Here, we also found SF3B1 transcriptome enriched with several metabolic process, suggesting a possible role for SF3B1 in metabolic programming in cancers. It is wellrecognized that SF3B1 regulate alternative splicing of several crucial genes involved in cell survival pathways implying its oncogenic potential in promoting tumorigenesis $^{24,40,41}$. Consistently, we found wide scale disruption of alternative splicing across the transcriptome, suggesting that SF3B1-driven alternative splicing has a pathogenic significance in endometrial cancer. Interestingly, we identified differential exon usage in one of the important small nucleolar RNAs (snoRNAs), the SNHG4. Previous studies demonstrated the overexpression of numerous snoRNAs in several cancers and their role in promoting proliferation, cell cycle progression, invasion, and metastasis ${ }^{45-47}$. Therefore, investigating the role of snoRNAs in endometrial cancer is quite intriguing; however, in-depth functional studies are required to dissect the role of such potential alternative spliced variants in endometrial cancer tumorigenesis.

Several lines of evidence suggest that PTEN, a tumor suppressor gene is frequently mutated in -30 to $50 \%$ of endometrial cancers ${ }^{48-50}$. Increased SF3B1 levels in tumors originated due to PTEN loss suggest that overexpression of SF3B1 coupled with PTEN loss might drive the endometrial cancer progression. However, more in-depth 
studies are required to test whether SF3B1 overexpression and PTEN loss are mutually exclusive in endometrial cancers. Importantly, these studies might provide molecular underpinnings of endometrial cancer initiation and progression.

Previous studies have shown that attenuation in splicing factor-driven RNA maturation promotes tumorigenesis through the accumulation of pre-mRNAs ${ }^{51,52}$. We found decreased expression of KSR2 due to impairment in SF3B1-mediated mRNA maturation and KSR2 partially contributes to SF3B1 pro-proliferative function. Given, multitude of genes impacted by mis-splicing due to splicing factor dysfunctions, the downstream functional impact is unlikely due to the altered splicing of a single gene variant. Instead, splicing factor deregulation may cause broad splicing aberrations resulting in induction of distinct transcriptional signature(s) that promote tumor progression. Thus, the partial contribution of KSR2 to overall SF3B1 function in our study indicates that SF3B1 exerts its functions through numerous downstream effector genes. For example, we found many of the SF3B1regulated genes associated with metabolic process (Supplementary Table 2). Therefore, future studies dovetailing transcriptomics and metabolomics may provide better understanding on the functional role of SF3B1 in endometrial cancer progression.

SF3B1 is a target of PLAD-B, a natural product with anti-tumor activity in both cancer cell lines and mouse xenograft models ${ }^{41,53,54}$. By impairing the binding of the spliceosome to the branch sequence, PLAD-B causes an accumulation of unspliced mRNA in cells, resulting in reduced viability ${ }^{35,55}$. Here, we found that PLAD-B decreased endometrial cancer cell proliferation, migration, and invasion in vitro. Furthermore, we found that PLAD-B reduced the endometrial cancer burden in our orthotopic endometrial cancer model, suggesting that PLAD-B, or a derivative, might be a viable therapeutic with which to treat endometrial cancer.

In summary, we report that SF3B1 plays an important role in endometrial cancer initiation and progression possibly through regulating KSR2 mRNA maturation. Thus, uncovering the precise role of SF3B1 in the progression of endometrial cancer will increase our knowledge of spliceosome function in endometrial cancer.

\footnotetext{
Acknowledgements

We thank Dr. Deborah J. Frank (Department of Obstetrics and Gynecology, Washington University) for assistance with manuscript editing and Marina N. Rowen and Gwen Kreker (Department of Obstetrics and Gynecology, Washington University) for technical assistance. We thank Dr. I. Sadaf Faroogi (University of Cambridge Metabolic Research Laboratories and NIHR Cambridge Biomedical Research Centre, Cambridge CB2 0QQ, UK) for kindly sharing pEGFPC1-hKSR2-WT and pEGFPC1 expression plasmids. We thank the Genome Engineering and iPSC Center (GEiC) at Washington University in St. Louis for SF3B1 exome sequencing in endometrial cancer cell lines. We also thank Genome Technology Access Center (GTAC) with transcriptome analysis. The Center is partially supported by $\mathrm{NCl}$ Cancer Center Support Grant
}

\#P30 CA91842 to the Siteman Cancer Center and by ICTS/CTSA Grant \#UL1TR002345 from the National Center for Research Resources (NCRR), a component of the National Institutes of Health $(\mathrm{NIH})$, and $\mathrm{NIH}$ Roadmap for Medical Research. This work was funded, in part, by National Institutes of Health/National Institute of Child Health and Human Development grants R01HD065435 and R00HD080742 to R.K. and Washington University School of Medicine start-up funds to R.K. and National Human Genome Research Institute (NHGRI) R00HG007940 to M.G. M.M.R. and M.G. were also supported by the V Foundation for Cancer Research under Award Number V2018-007.

\section{Author details}

'Department of Obstetrics and Gynecology, Center for Reproductive Health Sciences, Washington University School of Medicine, St. Louis, MO 63110, USA ${ }^{2}$ Division of Oncology, Department of Medicine, Washington University School of Medicine, St. Louis, MO 63110, USA. ${ }^{3}$ Genome Technology Access Center, McDonnell Genome Institute, Washington University School of Medicine, St. Louis, MO 63110, USA. ${ }^{4}$ Department of Obstetrics, Gynecology and Reproductive Biology, Michigan State University, Grand Rapids, MI 48824, USA ${ }^{5}$ Department of Genetics, Washington University School of Medicine, St. Louis, MO 63110, USA. ${ }^{6}$ Siteman Cancer Center, Washington University School of Medicine, St. Louis, MO 63110, USA. Division of Gynecologic Oncology, Department of Obstetrics and Gynecology, Washington University School of Medicine, St. Louis, MO 63110, USA

\section{Author contributions}

P.P. and R.K. designed experiments, conducted most of the studies, analyzed the data, and wrote the manuscript. S.B.C. assisted with critical animal surgeries, collected tissues, and generated some of the reagents. T.H.K. and E.T provided reagents and bioinformatics analysis, respectively. M.M.R., O.G., and M.G. performed alternative splicing analysis. R.K. conceived the project, supervised the work, and wrote the manuscript. All authors critically reviewed and edited the manuscript.

\section{Conflict of interest}

The authors declared that they have no conflict of interest.

\section{Publisher's note}

Springer Nature remains neutral with regard to jurisdictional claims in published maps and institutional affiliations.

Supplementary Information accompanies this paper at (https://doi.org/ 10.1038/s41419-020-03055-y).

Received: 7 May 2020 Revised: 21 September 2020 Accepted: 25 September 2020

Published online: 10 October 2020

\section{References}

1. Bell, D. W. \& Ellenson, L. H. Molecular genetics of endometrial carcinoma. Annu. Rev. Pathol. 14, 339-367 (2019).

2. Sheikh, M. A. et al. USA endometrial cancer projections to 2030: should we be concerned? Future Oncol. 10, 2561-2568 (2014).

3. Paronetto, M. P., Passacantilli, I. \& Sette, C. Alternative splicing and cell survival: from tissue homeostasis to disease. Cell Death Differ. 23, 1919-1929 (2016).

4. David, C. J. \& Manley, J. L. Alternative pre-mRNA splicing regulation in cancer: pathways and programs unhinged. Genes Dev. 24, 2343-2364 (2010).

5. Oltean, S. \& Bates, D. O. Hallmarks of alternative splicing in cancer. Oncogene 33, 5311-5318 (2014).

6. Srebrow, A. \& Kornblihtt, A. R. The connection between splicing and cancer. J. Cell Sci. 119, 2635-2641 (2006).

7. Zhou, X. et al. Splicing factor SRSF1 promotes gliomagenesis via oncogenic splice-switching of MYO1B. J. Clin. Invest. 129, 676-693 (2019).

8. Alsafadi, S. et al. Cancer-associated SF3B1 mutations affect alternative splicing by promoting alternative branchpoint usage. Nat. Commun. 7, 10615 (2016).

9. Visconte, V., Makishima, H., Maciejewski, J. P. \& Tiu, R. V. Emerging roles of the spliceosomal machinery in myelodysplastic syndromes and other hematological disorders. Leukemia 26, 2447-2454 (2012). 
10. Kahles, A. et al. Comprehensive analysis of alternative splicing across tumors from 8,705 patients. Cancer Cell 34, 211-224 e216 (2018).

11. Anczukow, O. \& Krainer, A. R. Splicing-factor alterations in cancers. RNA 22 1285-1301 (2016).

12. Bejar, R. Splicing factor mutations in cancer. Adv. Exp. Med. Biol. 907, 215-228 (2016).

13. Pellagatti, A. et al. Impact of spliceosome mutations on RNA splicing in myelodysplasia: dysregulated genes/pathways and clinical associations. Blood 132, 1225-1240 (2018)

14. Quesada, V. et al. Exome sequencing identifies recurrent mutations of the splicing factor SF3B1 gene in chronic lymphocytic leukemia. Nat. Genet. 44, 47-52 (2011).

15. Wang, L. et al. SF3B1 and other novel cancer genes in chronic lymphocytic leukemia. N. Engl. J. Med. 365, 2497-2506 (2011).

16. Yoshida, $\mathrm{K}$. et al. Frequent pathway mutations of splicing machinery in myelodysplasia. Nature 478, 64-69 (2011).

17. Zhang, J. et al. Disease-associated mutation in SRSF2 misregulates splicing by altering RNA-binding affinities. Proc. Natl Acad. Sci. USA 112, E4726-E4734 (2015).

18. Brooks, A. N. et al. A pan-cancer analysis of transcriptome changes associated with somatic mutations in U2AF1 reveals commonly altered splicing events. PLOS ONE 9, e87361 (2014).

19. Thol, F. et al. Frequency and prognostic impact of mutations in SRSF2, U2AF1, and ZRSR2 in patients with myelodysplastic syndromes. Blood 119, 3578-3584 (2012).

20. Seiler, M. et al. Somatic mutational landscape of splicing factor genes and their functional consequences across 33 cancer types. Cell Rep. 23, 282-296. e284 (2018).

21. Chang, Y. S., Huang, H. D., Yeh, K. T. \& Chang, J. G. Genetic alterations in endometrial cancer by targeted next-generation sequencing. Exp. Mol. Pathol. 100, 8-12 (2016).

22. Wahl, M. C., Will, C. L. \& Luhrmann, R. The spliceosome: design principles of a dynamic RNP machine. Cell 136, 701-718 (2009).

23. Cretu, C. et al. Molecular architecture of SF3b and structural consequences of its cancer-related mutations. Mol. Cell 64, 307-319 (2016).

24. Jimenez-Vacas, J. M. et al. Spliceosome component SF3B1 as novel prognostic biomarker and therapeutic target for prostate cancer. Transl. Res. 212, 89-103 (2019).

25. Dolatshad, $\mathrm{H}$. et al. Disruption of SF3B1 results in deregulated expression and splicing of key genes and pathways in myelodysplastic syndrome hematopoietic stem and progenitor cells. Leukemia 29, 1092-1103 (2015).

26. Zhang, L. et al. Knockdown of SF3B1 inhibits cell proliferation, invasion and migration triggering apoptosis in breast cancer via aberrant splicing. Breast Cancer. https://doi.org/10.1007/s12282-020-01045-8 (2020).

27. Xiao, X. Y. et al. Licochalcone $A$ inhibits growth of gastric cancer cells by arresting cell cycle progression and inducing apoptosis. Cancer Lett. 302, 69-75 (2011).

28. Keirsebilck, A. et al. Molecular cloning of the human p120ctn catenin gene (CTNND1): expression of multiple alternatively spliced isoforms. Genomics $\mathbf{5 0}$, 129-146 (1998).

29. Pino, M. S. et al. Human Mena+11a isoform serves as a marker of epithelial phenotype and sensitivity to epidermal growth factor receptor inhibition in human pancreatic cancer cell lines. Clin. Cancer Res. 14, 4943-4950 (2008).

30. Shapiro, I. M. et al. An EMT-driven alternative splicing program occurs in human breast cancer and modulates cellular phenotype. PLOS Genet. 7, e1002218 (2011)

31. Warzecha, C. C., Sato, T. K., Nabet, B., Hogenesch, J. B. \& Carstens, R. P. ESRP1 and ESRP2 are epithelial cell-type-specific regulators of FGFR2 splicing. Mol. Cell 33, 591-601 (2009).

32. Sakai, T. et al. Pladienolides, new substances from culture of Streptomyces platensis Mer-11107. I. Taxonomy, fermentation, isolation and screening. J. Antibiot. (Tokyo) 57, 173-179 (2004)
33. Sakai, T., Asai, N., Okuda, A., Kawamura, N. \& Mizui, Y. Pladienolides, new substances from culture of Streptomyces platensis Mer-11107. II. Physicochemical properties and structure elucidation. J. Antibiot. (Tokyo) 57, 180-187 (2004).

34. Martinez-Montiel, N., Rosas-Murrieta, N. H., Martinez-Montiel, M., GasparianoCholula, M. P. \& Martinez-Contreras, R. D. Microbial and natural metabolites that inhibit splicing: a powerful alternative for cancer treatment. Biomed. Res. Int. 2016, 3681094 (2016).

35. Kotake, Y. et al. Splicing factor SF3b as a target of the antitumor natural product pladienolide. Nat. Chem. Biol. 3, 570-575 (2007).

36. Sato, $M$. et al. High antitumor activity of pladienolide $B$ and its derivative in gastric cancer. Cancer Sci. 105, 110-116 (2014).

37. Fernandez, M. R., Henry, M. D. \& Lewis, R. E. Kinase suppressor of Ras 2 (KSR2) regulates tumor cell transformation via AMPK. Mol. Cell Biol. 32, 3718-3731 (2012).

38. Dougherty, M. K. et al. KSR2 is a calcineurin substrate that promotes ERK cascade activation in response to calcium signals. Mol. Cell 34, 652-662 (2009).

39. Frodyma, D., Neilsen, B., Costanzo-Garvey, D., Fisher, K. \& Lewis, R. Coordinating ERK signaling via the molecular scaffold kinase suppressor of Ras. F1000Research 6, 1621 (2017).

40. Zhang, Y. et al. Inhibition of splicing factor $3 b$ subunit 1 (SF3B1) reduced cell proliferation, induced apoptosis and resulted in cell cycle arrest by regulating homeobox A10 (HOXA10) splicing in AGS and MKN28 human gastric cancer cells. Med. Sci. Monit. 26, e919460 (2020).

41. Zhang, Q. et al. Inhibition of SF3b1 by pladienolide B evokes cycle arrest, apoptosis induction and p73 splicing in human cervical carcinoma cells. Artif. Cells Nanomed. Biotechnol. 47, 1273-1280 (2019).

42. Saldi, T., Cortazar, M. A., Sheridan, R. M. \& Bentley, D. L. Coupling of RNA polymerase II transcription elongation with pre-mRNA aplicing. J. Mol. Biol. 428, 2623-2635 (2016).

43. Liu, H., Luo, M. \& Wen, J. K. mRNA stability in the nucleus. J. Zhejiang Univ. Sci. B 15, 444-454 (2014).

44. Dalton, W. B. et al. Hotspot SF3B1 mutations induce metabolic reprogramming and vulnerability to serine deprivation. J. Clin. Invest. 130, 4708-4723 (2019).

45. Zimta, A. A. et al. An emerging class of long non-coding RNA with oncogenic role arises from the snoRNA host genes. Front. Oncol. 10, 389 (2020).

46. Liao, J. et al. Small nucleolar RNA signatures as biomarkers for non-small-cell lung cancer. Mol. Cancer 9, 198 (2010).

47. Gong, J. et al. A Pan-cancer analysis of the expression and clinical relevance of small nucleolar RNAs in human cancer. Cell Rep. 21, 1968-1981 (2017).

48. Simpkins, S. B., Peiffer-Schneider, S., Mutch, D. G., Gersell, D. \& Goodfellow, P. J. PTEN mutations in endometrial cancers with $10 \mathrm{q} \mathrm{LOH}$ : additional evidence for the involvement of multiple tumor suppressors. Gynecol. Oncol. 71, 391-395 (1998).

49. Cheng, $\mathrm{H}$. et al. A genetic mouse model of invasive endometrial cancer driven by concurrent loss of Pten and Lkb1 is highly responsive to mTOR inhibition. Cancer Res. 74, 15-23 (2014).

50. Daikoku, T. et al. Conditional loss of uterine Pten unfailingly and rapidly induces endometrial cancer in mice. Cancer Res. 68, 5619-5627 (2008).

51. Ding, $K$. et al. RNA splicing factor USP39 promotes glioma progression by inducing TAZ mRNA maturation. Oncogene 38, 6414-6428 (2019).

52. Huang, $Y$. et al. Overexpression of USP39 predicts poor prognosis and promotes tumorigenesis of prostate cancer via promoting EGFR mRNA maturation and transcription elongation. Oncotarget 7, 22016-22030 (2016).

53. Yokoi, A. et al. Biological validation that SF3b is a target of the antitumor macrolide pladienolide. FEBS J. 278, 4870-4880 (2011).

54. Aouida, M., Eid, A. \& Mahfouz, M. M. CRISPR/Cas9-mediated target validation of the splicing inhibitor Pladienolide B. Biochim. Open 3, 72-75 (2016).

55. Effenberger, K. A. et al. Coherence between cellular responses and in vitro splicing inhibition for the anti-tumor drug pladienolide B and its analogs. J. Biol. Chem. 289, 1938-1947 (2014). 